\title{
Review Article \\ HINT1 in Neuropsychiatric Diseases: A Potential Neuroplastic Mediator
}

\author{
Peng Liu, ${ }^{1}$ Zhongwei Liu, ${ }^{2}$ Jiabei Wang, ${ }^{3}$ Xiancang Ma, ${ }^{4}$ and Yonghui Dang ${ }^{1}$ \\ ${ }^{1}$ College of Medicine \& Forensics, Key Laboratory of the Health Ministry for Forensic Medicine, \\ Key Laboratory of Environment and Genes Related to Diseases of the Education Ministry, \\ Xi'an Jiaotong University Health Science Center, Xi'an 710061, China \\ ${ }^{2}$ Department of Cardiology, Shaanxi Provincial People's Hospital, Xi'an 710068, China \\ ${ }^{3}$ Department of Pharmaceutical Sciences, School of Pharmacy, University of Maryland Baltimore, Baltimore MD 21201, USA \\ ${ }^{4}$ Department of Psychiatry, First Affiliated Hospital of Xi'an Jiaotong University Health Science Center, Xi'an, China
}

Correspondence should be addressed to Yonghui Dang; psydyh@mail.xjtu.edu.cn

Received 14 April 2017; Revised 23 August 2017; Accepted 18 September 2017; Published 30 October 2017

Academic Editor: Bingjin Li

Copyright ( 2017 Peng Liu et al. This is an open access article distributed under the Creative Commons Attribution License, which permits unrestricted use, distribution, and reproduction in any medium, provided the original work is properly cited.

\begin{abstract}
Although many studies have investigated the functions of histidine triad nucleotide-binding protein 1 (HINT1), its roles in neurobiological processes remain to be fully elucidated. As a member of the histidine triad (HIT) enzyme superfamily, HINT1 is distributed in almost every organ and has both enzymatic and nonenzymatic activity. Accumulating clinical and preclinical evidence suggests that HINT1 may play an important role as a neuroplastic mediator in neuropsychiatric diseases, such as schizophrenia, inherited peripheral neuropathies, mood disorders, and drug addiction. Though our knowledge of HINT1 is limited, it is believed that further research on the neuropathological functions of HINT1 would eventually benefit patients with neuropsychiatric and even psychosomatic diseases.
\end{abstract}

\section{Introduction to HINT1}

Proteins containing the histidine triad (HIT) motif, a conserved HisXHisXHis sequence (in which X represents any hydrophobic amino acid), constitute an enzyme superfamily known as the HIT proteins [1]. According to enzyme activity classification, HIT proteins can be classified into three branches: nucleoside phosphoramidate hydrolases, dinucleotide hydrolases, and nucleotidylyl transferases. HIT proteins are conserved throughout evolution, and more than 35 members of this superfamily have been found in 29 species, including bacteria, archaea, yeast, plants, $C$. elegans, Drosophila, and mammals, implying that HINT1 exerts basic and essential physiological functions [2]. The human genome encodes seven HIT proteins, which mainly serve as nucleoside transferases and hydrolases and can be divided into five classes: histidine triad nucleotide-binding protein (HINT), galactosyl-1-phosphate uridine acyltransferase, aprataxin, DCPS/DCS-1, and the brittle histidine triad proteins [3-6].
The histidine triad nucleotide-binding protein (HINT) (including human HINT and nonhuman Hint) is the first class of HIT superfamily. It is now suggested that at least one HINT gene is thought to exist in all sequenced genomes. Three independent HINT genes encoding the HINT1, HINT2, and HINT3 proteins are found in the human genome. Genes encoding HINT1 proteins are localized on human chromosome 5q31.2, with a full length of $6160 \mathrm{bp}$, containing three exons. HINT1 mRNA is composed of $782 \mathrm{bp}$, encoding a 126-amino acid cytosolic protein molecule with a relative molecular mass of approximately $14 \mathrm{kDa}$ (Figure 1) [3, 7]. According to nuclear magnetic resonance (NMR) and crystallography studies, HINT1 is one of the purine nucleotide-binding proteins. Two subunits constitute a homodimer structure with a binding site for purine bases and a binding site for ribose on each subunit (Figure 2) $[1,8,9]$.

HINT1 was first described as a protein kinase inhibitor in 1990 [10] and supposed to be protein kinase C inhibitor 1 (PKCI-1) in early literature $[11,12]$. Although direct or 


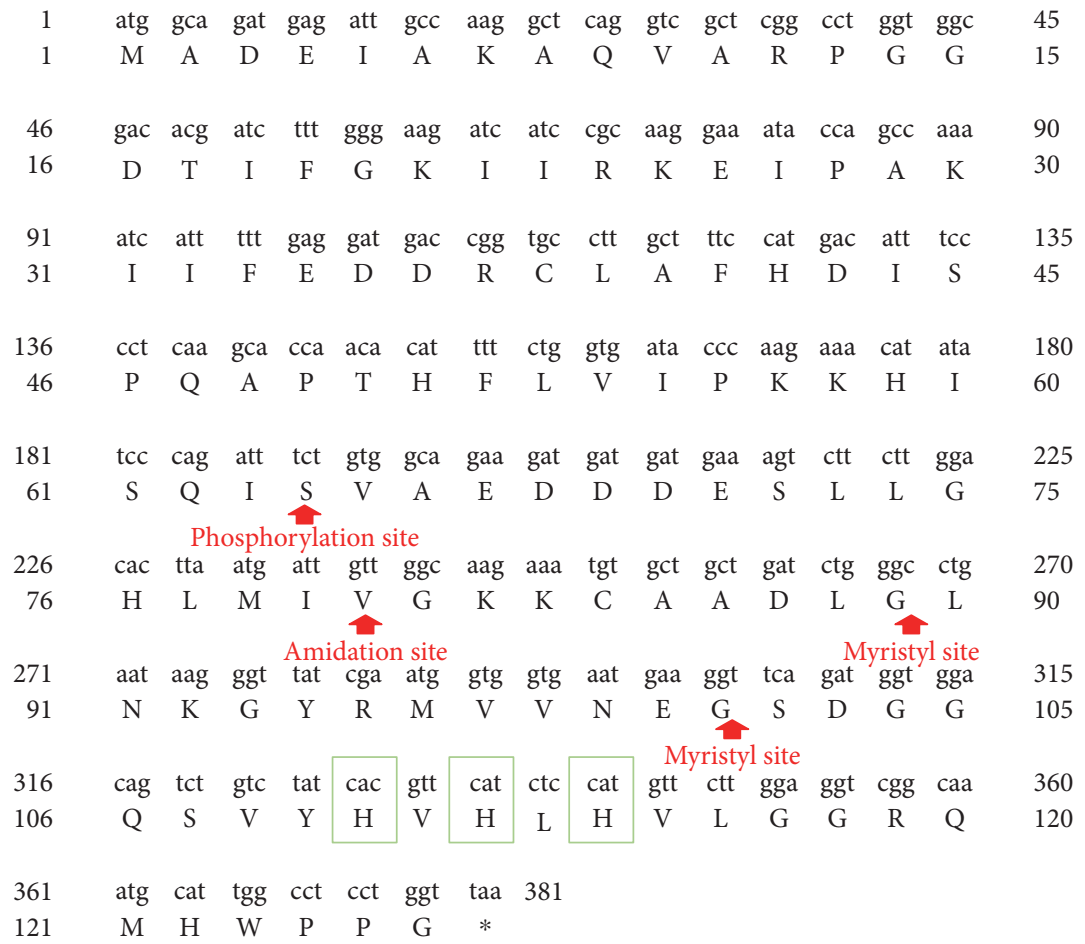

FIGURE 1: DNA and protein sequences of human HINT1. Green boxes indicate the histidine triad (HIT) domain. The potential modification sites of the protein are shown, including the phosphorylation site, amidation site, and myristyl site.

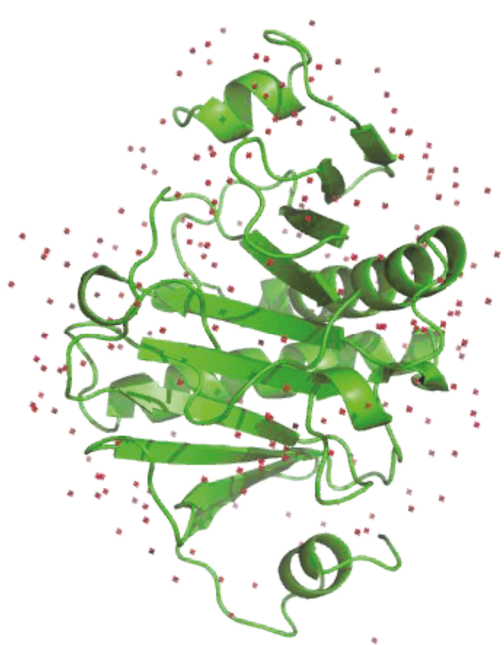

FIGURE 2: Crystal diffraction pattern of the HINT1 protein. HINT1 consists of a homodimer, each subunit of which contains two $\alpha$-helices and three $\beta$-sheets. From http://www.rcsb.org/pdb.

indirect interactions between HINT1 and protein kinase $\mathrm{C}$ (PKC) were suggested, the inhibitory effects of HINT1 on PKC are still doubtful. Therefore, PKCI-1 was renamed to HINT1 [1]. The abbreviation PKCI is still occasionally used in the current literature with the altered meaning of protein kinase $\mathrm{C}$ interacting protein 1 , which was indicated in Klein et al.'s study [13].

\section{HINT1 in Tumorigenesis}

Although HINT1 is known for its enzyme activity, studies have shown that it has additional functions unrelated to its enzymatic activity [14], such as tumorigenesis suppression $[15,16]$. Accumulating evidence indicates that HINT1 plays a role as a haploinsufficient tumor suppressor in multiple malignant diseases, but little is known about the mechanism $[15,16]$. Several recent studies demonstrated that by inhibiting the activities of transcription factors including AP1 [17], TFIIH [18], MITF [19], and USF2 [20], HINT1 could regulate gene expression in the $\mathrm{Wnt} /$ beta-catenin signaling pathway [21]. Therefore, it seems that HINT1 may exert potential anticancer effects as a gene transcriptional regulator. Currently, studies are focusing on the clinical relevance of HINT1 expression in several human specific cancers [22-24].

\section{HINT1 in Neuropsychiatric Disorders}

HINT1 has a wide range of distribution in various tissues including liver, kidney, stomach, and brain in humans and rodents [13]. HINT1 is expressed in the central nervous system (CNS) of mice and is particularly enriched in the olfactory sensory system, cerebral cortex, hippocampus, part of the thalamus, hypothalamus, midbrain, pons, and medulla oblongata [25]. The distribution of HINT1 in the CNS provides anatomical evidence for its potential importance in neuronal function. 
3.1. Schizophrenia. The HINT1 gene is located on a genetic locus highly associated with schizophrenia $(5 q 31.2)[26,27]$. Schizophrenia is a common psychiatric disease with manifestations of positive symptoms (hallucinations, delusions, disorganized speech, disorganized behavior, catatonic behavior, agitation, etc.) and negative symptoms (blunted affect, emotional withdrawal, apathetic social withdrawal, stereotyped thinking, attentional impairment, etc.), as well as cognitive, affective, and aggressive symptoms [28]. The etiology of schizophrenia is complicated, including epigenetic changes and interactions between genetic susceptibility and environment [29].

Vawter et al. found that HINT1 was significantly decreased in the dorsolateral prefrontal cortex (DLPFC) and prefrontal cortex in patients with schizophrenia [30-32]. Notably, the HINT1 gene is located in the SPEC2/PDZGEF2/ACSL6 region of 5q22-23, which is associated with schizophrenia [33]. The same team then evaluated eight single nucleotide polymorphisms (SNPs) in the HINT1 gene in Irish study of high-density schizophrenia families (ISHDSF, 1350 subjects and 273 pedigrees) and Irish case-control study of schizophrenia (ICCSS, 655 patients and 626 controls). They further compared expression levels of HINT1 in postmortem brain samples provided by the Stanley Medical Research Institute and concluded that mutations in the HINT1 gene were potentially correlated with schizophrenia [7]. Varadarajulu et al. [34] found that the expression of HINT1 protein was upregulated in the thalamus but downregulated in the DLPFC in postmortem brain samples of patients with schizophrenia compared to those of healthy controls, consistent with results from another study in 2011 [35]. Additionally, findings from the abovementioned studies suggest that the association between HINT1 and schizophrenia is gender-specific and may only exist in male patients $[7,32,33]$.

The results obtained from clinical studies are further supported by studies of HINT1 knockout (KO) mice. Barbier and colleagues [36] demonstrated that compared with wild-type (WT) mice, HINT1 KO mice were more sensitive to acute amphetamine- (AMPH-) induced hyperlocomotor behavior. Quantitative microdialysis of the kinetics of dopamine (DA) in the striatum or nucleus accumbens (NAc) showed that presynaptic DA neurotransmission in these regions did not underlie the AMPH-induced behavioral phenotype of KO mice. However, systemic administration of apomorphine, a dopamine receptor agonist, significantly increased $\mathrm{KO}$ mouse locomotor activity, suggesting that the postsynaptic DA transmission may be dysregulated in $\mathrm{KO}$ mice. Considering that schizophrenia is often accompanied by dopaminergic system hyperfunction [37] and the hyperactivity induced by $\mathrm{AMPH}$ represents the positive symptom-like behavior in rodent models for schizophrenia [38], HINT1 KO mice appear to be a useful genetic animal model for studying schizophrenia. Furthermore, we found that HINT1 plays a role in a social isolation (SI) mouse model, characterized by behavioral abnormalities similar to those in schizophrenia, and potential interactions among HINT1, N-methyl$\mathrm{D}$-aspartate receptor (NMDAR), and DA type 2 receptor
(D2R) may underlie the schizophrenia-like behavioral deficits induced by SI $[39,40]$.

3.2. Inherited Peripheral Neuropathies (IPNs). IPNs, which affect the peripheral nervous system (PNS), are neuromuscular and neurodegenerative disorders characterized by disrupted communication between the CNS and body. As one of the most common inherited neuromuscular disorders, the prevalence of IPNs is approximately 1 in 2500 [41]. IPNs include a large group of disorders involving multiple genes and complex phenotypes, so the correct diagnosis of each genetic subtype is a thorny problem for clinicians. At present, more than 100 different subtypes of IPNs have been identified, each with its own specific clinical features, pathophysiology, and prognosis. The unidentified mutations make it difficult to apply molecular diagnosis, and therefore, clinical features and developmental patterns are currently used to direct identification of genetic subtypes in patients with IPNs.

One study showed that mutations of HINT1 may be a cause of distal hereditary motor neuropathies [42]. In addition, Zimoń et al. [43] identified eight different mutations of the HINT1 gene in a cohort of 50 autosomal recessive axonal neuromyotonia (ARAN) patients with neuromyotonia (NM) from 33 unrelated nuclear families. NM is characterized by delayed muscular relaxation after voluntary contractions, induced by overexcited motor axons in the PNS [44]. In order to analyze the association between HINT1 and ARAN patients with NM, Zimon and colleagues [43] screened patients and found a mutation rate at $11 \%$ in irrelevant patients with autosomal recessive peripheral neuropathy, which was $76 \%$ in ARAN patients with NM. Thus, there is a robust causal genetic association between HINT1 and ARAN patients with NM. However, Horga et al. did not detect variation of the HINT1 gene by direct sequencing of 152 patients with IPNs in England and Spain, indicating a regional specificity in this association [45-47].

Zimon and colleagues also evaluated the expression levels of HINT1 in mouse tissues, such as heart, lung, and liver [43]. The results showed that HINT1 was enriched in the sciatic nerve in mice, indicating that HINT1 is a vital component of the function of PNS. Furthermore, they implemented in vivo genetic complementation analysis by using HINT1 deficit yeast strain (BY8-5c from Saccharomyces cerevisiae strain) and then analyzed HINT1 expression levels in lymphoblastoid cell cultures from affected individuals and irrelevant controls, respectively [43], identifying that mutations of HINT1 belong to loss-of-function mutations. Thus, a new genetic subtype was defined based on this functional mutation, namely, autosomal recessive axonal neuropathy with neuromyotonia (ARAN-NM) [43]. Even so, by using knockout mice, Seburn and colleagues demonstrated that HINT1 knockout mice may be useful for studying the biochemical activities of HINT1, but these mice do not provide a disease model or a means for investigating the basis of HINT1associated neuropathy and neuromyotonia [48]. Therefore, further investigation is needed to determine whether HINT1 functions are species-specific. 
3.3. Mood Disorders. Mood disorder is featured by obvious and sustained episodes of mania or depression with the clinical manifestations of major depressive disorder (MDD) and bipolar disorder (BP) [49].

Elashoff et al. [50] performed a meta-analysis of 12 microarray studies and concluded that expression of HINT1 was decreased in postmortem brains of patients with BP. A study using HINT1 KO mice demonstrated that KO mice showed decreased depression-like behavior and enhanced cognitive ability. Additionally, KO mice showed abnormalities in the tail suspension test (TST), which could be alleviated by acute administration of the mood-stabilizer valproic acid (VPA) [51]. Increased corticosterone secretion in HINT1 KO mice was also observed [51]. These behavioral and endocrine changes indicate that HINT1 participates in emotional regulation in the CNS, and its absence may lead to manic-like behavior. Furthermore, another study using HINT1 KO mice suggested HINT1 KO mice exhibited behavioral and molecular alterations paralleling those described in BP patients. Thus, HINT1 KO mice could be used as an appropriate model for studying BP and may help identify novel targets and drugs to treat this mental disorder [52].

Interestingly, Martins-de-Souza et al. [53] screened differential protein expressions in the DLPFC of postmortem brains from 24 patients with MDD and 12 controls and detected increased expression of HINT1 in patients with MDD without psychotic symptoms. Moreover, in a study using the chronic mild stress (CMS) depression model to explore the antidepressant effect of oleamide, proteomics analysis showed that the expression level of HINT1 protein in the hippocampus of the CMS group was increased [54]. These results indicate that in different episodes of mood disorders, HINT1 works exactly the opposite.

3.4. Anxiety Disorder. There is currently a shortage of clinical studies on the association between HINT1 and anxiety disorder, and results from preclinical studies are not consistent. Barbier et al. [36] conjectured that anxiolytic-like behaviors were included in HINT1 deficiency-induced emotional alterations [51]. While Varadarajulu et al. studied the behaviors of male HINT1 KO mice in a battery of tests. They concluded that HINT1 KO mice exhibited increased anxiety-like behavior compared with that in WT mice [55]. What is more, Jackson et al. [56] found that in male HINT1 KO mice, the acute administration of nicotine resulted in production of anxietylike responses rather than its anxiolytic effects, and administration of diazepam failed to induce anxiolytic responses. However, the anxiety-like behaviors described above were not observed in female HINT1 KO mice, further supporting the aforementioned existence of gender differences in the behavioral impact of HINT1. All results from the anxiety studies were controversial, probably because of deviations in methods, experimental equipment, and animal age (e.g., Wang et al. often use older animals than Varadarajulu et al.).

3.5. Pain and Analgesia. The human $\mu$-opioid receptor (MOR), a G protein-coupled receptor (GPCR), is the molecular target of morphine-induced analgesia and opiate-related addiction. Guang et al. [57] first discovered the specific interaction between HINT1 and the C-terminus of human MOR using a yeast two-hybrid system. This interaction reduced the desensitization and phosphorylation of MOR. Meanwhile, increased basic pain threshold and enhanced morphine-induced analgesic effects were found in HINT1 $\mathrm{KO}$ mice. However, the dose-response curve indicated that KO mice exhibited a greater extent of tolerance to morphine-induced analgesia than WT mice. In addition, our group and Garzon's research team revealed that HINT1 deficiency could induce abnormalities in the hot-plate test, formalin-induced inflammatory pain, and CCI-induced neuropathic nociception [58-60]. In particular, Garzon and colleagues demonstrated that the inhibitor of HINT1 enzymatic activity, guanosine- $5^{\prime}$-tryptamine carbamate (TpGc), significantly enhanced morphine antinociception and alleviated mechanical allodynia but prevented the development of tolerance to opioids [61]. These results show the negative regulatory effect of HINT1 in MOR-mediated morphine-induced analgesia. However, an association study of 2294 patients with cancer pain did not find a correlation between SNP mutations in the HINT1 gene and opioid dose [62].

3.6. Drug Addiction. Association analysis from two independent samples indicates that mutations in the HINT1 gene are associated with phenotypes of nicotine dependence. Further analysis of mRNA expression in human postmortem brain showed that smoking status and phenotype were associated with HINT1 expression [63]. Chronic nicotine administration elevated HINT1 expression in mouse NAc, which could then be reversed by a nicotine antagonist, mecamylamine, after 24 hours or drug withdrawal after 72 hours [63]. These results show a genetic association between HINT1 and nicotine dependence. Jackson et al. [64] employed the conditioned place preference (CPP) reward test and conditioned place aversion (CPA) test to evaluate emotional and somatic symptoms after nicotine withdrawal. Significant CPA after withdrawal was found in both HINT1 KO and WT mice. In HINT1 KO mice, however, nicotine failed to induce significant CPP and somatic withdrawal symptoms (e.g., hyperalgesia) were alleviated. This study could further support the conclusion that HINT1 plays a role in regulating behaviors associated with nicotine reward and withdrawal. However, in an open-label randomized trial of nicotine replacement therapy (NRT) covering 374 nicotine-dependent smokers, the results do not support the relationship between HINT1 gene mutation and smoking cessation [65].

Relatively few studies have examined the role of HINT1 in addiction induced by other abused drugs. Romanova et al. [66] found that after a single injection of cocaine, HINT1 peak intensities increased significantly in the medial prefrontal cortex (mPFC) of low cocaine responder (LCRs) rats in the open field test. Previous studies showed that the LCRs were more sensitive to cocaine-induced behavioral sensitization compared to high cocaine responders (HCRs) $[67,68]$. Increased cocaine CPP [69] and self-administration motivation [70] exhibited by LCRs suggests that LCRs are sensitive to cocaine addiction. Thus, HINT1 is highly expressed in the susceptible phenotype of cocaine addiction. 


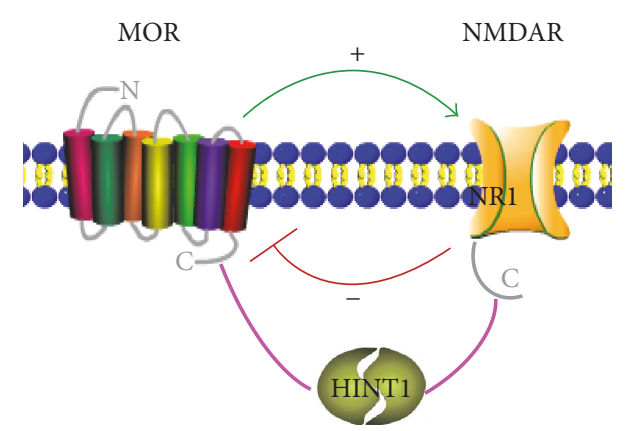

(a)

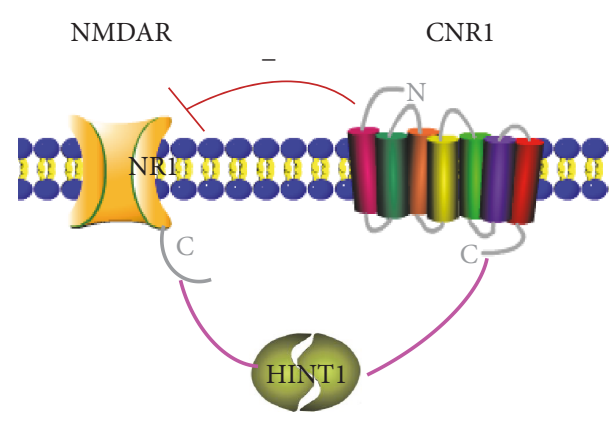

(b)

FIGURE 3: The pattern of HINT1 interacting with GPCRs. (a) HINT1 interacts with the C-terminus of $\mu$-opioid receptor (MOR). HINT1 also interacts with the NR1 subunit of NMDAR. To prevent opioids from producing an excessive reduction of neuronal excitability, NMDARs are recruited to the MOR environment, where they become activated to restrain opioid signaling. In this context, HINT1 stabilizes the functional interaction between MOR and NMDAR. (b) HINT1 may also associate with cannabinoid receptor type I (CNR1). CNR1 can negatively regulate NMDAR function when the receptor is coupled to HINT1.

Our recent study has demonstrated that the HINT1 protein, particularly in the NAc, also plays a vital role in methamphetamine-induced CPP [71].

3.7. Down's Syndrome (DS). Weitzdoerfer et al. [72] used two-dimensional gel electrophoresis and mass spectrometry to analyze proteins in cortical tissue from aborted human fetus. They found that different kinds of early life proteins, including HINT1, that participate in neural differentiation, neural migration, and synaptic transmission were deficient in DS.

3.8. Brain Aging. Brain aging is one of the major high risk factors for many neurodegenerative disorders such as Alzheimer's disease (AD). Nevertheless, the molecular mechanisms of brain aging are complicated and still unclear. Rassoul et al. [73] analyzed differential transcriptome expression in the temporal cortex of the primate Microcebus murinus. Of 695 different genes identified among young healthy animals, old healthy animals, and AD-like animals, approximately $1 / 3$ showed the same expression changes in healthy aging animals and AD-like animals, including the downregulation of HINT1 and HINT2. These findings indicate the possible contribution of HINT1 in the biological process of brain aging.

\section{Potential Role of HINT1 in Neuroplasticity}

As reviewed thus far, HINT1 is implicated in diverse neurological and neuropsychiatric diseases. Related to the latter, our studies have revealed that HINT1 is involved in SI mice model, which could induce behavioral abnormalities related to the core symptoms of certain neuropsychiatric disorders $[39,40]$. Neuropsychiatric disorders are a class of diseases closely related to the environment and genetics. One of the core problems in neuropsychiatric disorders is abnormal changes in neuroplasticity [74]. Therefore, it could be hypothesized that HINT1 may play an important role related to neuroplasticity in neuropsychiatric disorders. Thus, HINT1 is a potential promising neuroplasticity mediator in neuropsychiatric diseases.
Actually, on one hand, HINT1 could trigger apoptosis independent of its enzymatic activity [14], while there is little research on the exact role of HINT1 in apoptosis. On the other hand, a growing body of evidence suggests that HINT1 acts as a molecular switch regulating the interaction and functional association between GPCRs and NMDARs. For example, HINT1 could stabilize the interaction between MOR/cannabinoid receptor type 1 (CNR1) and NMDARs, promoting (e.g., MOR) or reducing (e.g., CNR1) its glutamatergic activity (Figure 3 ) [57, 59, 60, 75-83]. HINT1 protein may also participate in conveying information mediated by GPCRs to different signaling pathways, especially the glutamate NMDAR-mediated neurotransmission and functional neural plasticity, such as long-term potentiation (LTP) $[60,76,84]$. Moreover, our accepted study indicated that under both basal and chronic immobilization stress conditions, compared to WT mice, HINT1 KO mice expressed more hippocampal BDNF [85], which is also a key molecule engaged in neuroplasticity [86, 87]. However, to understand the specific role of HINT1 in neuroplasticity, more in-depth study is needed.

\section{Summary and Prospect}

Since HINT1 was discovered to be involved in a variety of biological phenomena, the research interest in this protein has been increasing. Though many studies have aimed to elucidate its roles in cell physiology, the complete range of functions of HINT1 is yet to be determined. The known functions of HINT1, such as tumor suppression, nucleoside transferase, and hydrolase functions, are only a tiny fraction of the whole picture. Currently, treatments for human neuropsychiatric diseases rely on a very limited selection of drugs and therapies, primarily because of our superficial knowledge of the pathogenesis of these diseases. Reviewing the available literature on HINT1, we found that HINT1 is highly related to many neuropsychiatric diseases including schizophrenia, mood disorder, drug addiction, and so on, and HINT1 may participate in neuropsychiatric diseases as a potential neuroplastic mediator. While many studies describe the correlation 
between HINT1 and neuropsychiatric diseases, few of them describe specific mechanisms. Thus, further study of HINT1 would be of potential value for expanding basic research, diagnosis, and treatment of neuropsychiatric and even psychosomatic diseases.

\section{Conflicts of Interest}

The authors declare that they have no conflicts of interest.

\section{Acknowledgments}

This research is supported by grants from the National Science Foundation of China (no. 81771435, no. 81371473, and no. 81171262), Natural Science Basic Research Plan in Shaanxi Province of China (no. 2016JM8078), and 2017 Open Research Fund of State Key Laboratory of Mechanical Manufacturing Systems Engineering (no. sklms2017002).

\section{References}

[1] C. Brenner, P. Garrison, J. Gilmour et al., "Crystal structures of HINT demonstrate that histidine triad proteins are GalTrelated nucleotide-binding proteins," Nature Structural Biology, vol. 4, no. 3, pp. 231-238, 1997.

[2] B. Seraphin, "The HIT protein family: a new family of proteins present in prokaryotes, yeast and mammals," DNA Sequence, vol. 3, no. 3, pp. 177-179, 1992.

[3] C. Brenner, "Hint, Fhit, and GalT: function, structure, evolution, and mechanism of three branches of the histidine triad superfamily of nucleotide hydrolases and transferases," Biochemistry, vol. 41, no. 29, pp. 9003-9014, 2002.

[4] A. W. Kijas, J. L. Harris, J. M. Harris, and M. F. Lavin, "Aprataxin forms a discrete branch in the HIT (histidine triad) superfamily of proteins with both DNA/RNA binding and nucleotide hydrolase activities," Journal of Biological Chemistry, vol. 281, no. 20, pp. 13939-13948, 2006.

[5] D. A. Kwasnicka, A. Krakowiak, C. Thacker, C. Brenner, and S. R. Vincent, "Coordinate expression of NADPH-dependent flavin reductase, Fre-1, and Hint-related 7meGMP-directed hydrolase, DCS-1," Journal of Biological Chemistry, vol. 278, no. 40, pp. 39051-39058, 2003.

[6] H. Liu, N. D. Rodgers, X. Jiao, and M. Kiledjian, "The scavenger mRNA decapping enzyme DcpS is a member of the HIT family of pyrophosphatases," The EMBO Journal, vol. 21, no. 17, pp. 4699-4708, 2002.

[7] Q. Chen, X. Wang, F. A. O’Neill, D. Walsh, K. S. Kendler, and $\mathrm{X}$. Chen, "Is the histidine triad nucleotide-binding protein 1 (HINT1) gene a candidate for schizophrenia?," Schizophrenia Research, vol. 106, no. 2-3, pp. 200-207, 2008.

[8] G. Bai, B. Feng, J. B. Wang, E. Pozharski, and M. Shapiro, "Studies on ligand binding to histidine triad nucleotide binding protein 1," Bioorganic \& Medicinal Chemistry, vol. 18, no. 18, pp. 6756-6762, 2010.

[9] J. Gilmour, N. Liang, and J. M. Lowenstein, "Isolation, cloning and characterization of a low-molecular-mass purine nucleoside- and nucleotide-binding protein," Biochemical Journal, vol. 326, no. 2, pp. 471-477, 1997.

[10] J. D. Pearson, D. B. DeWald, W. R. Mathews et al., “Amino acid sequence and characterization of a protein inhibitor of protein kinase C," The Journal of Biological Chemistry, vol. 265, no. 8, pp. 4583-4591, 1990.

[11] K. Huebner, J. C. Saldivar, J. Sun, H. Shibata, and T. Druck, "Hits, Fhits and Nits: beyond enzymatic function," Advances in Enzyme Regulation, vol. 51, no. 1, pp. 208-217, 2011.

[12] J. Martin, M. V. St-Pierre, and J. F. Dufour, "Hit proteins, mitochondria and cancer," Biochimica et Biophysica Acta (BBA) - Bioenergetics, vol. 1807, no. 6, pp. 626-632, 2011.

[13] M. G. Klein, Y. Yao, E. D. Slosberg, C. D. Lima, Y. Doki, and I. B. Weinstein, "Characterization of PKCI and comparative studies with FHIT, related members of the HIT protein family," Experimental Cell Research, vol. 244, no. 1, pp. 2632, 1998.

[14] J. Weiske and O. Huber, "The histidine triad protein Hint1 triggers apoptosis independent of its enzymatic activity," Journal of Biological Chemistry, vol. 281, no. 37, pp. 2735627366, 2006.

[15] H. Li, Y. Zhang, T. Su, R. M. Santella, and I. B. Weinstein, "Hint1 is a haplo-insufficient tumor suppressor in mice," Oncogene, vol. 25, no. 5, pp. 713-721, 2006.

[16] T. Su, M. Suzui, L. Wang, C. S. Lin, W. Q. Xing, and I. B. Weinstein, "Deletion of histidine triad nucleotide-binding protein 1/PKC-interacting protein in mice enhances cell growth and carcinogenesis," Proceedings of the National Academy of Sciences of the United States of America, vol. 100, no. 13, pp. 7824-7829, 2003.

[17] L. Wang, Y. Zhang, H. Li, Z. Xu, R. M. Santella, and I. B. Weinstein, "Hint1 inhibits growth and activator protein-1 activity in human colon cancer cells," Cancer Research, vol. 67, no. 10, pp. 4700-4708, 2007.

[18] N. Korsisaari and T. P. Makela, "Interactions of Cdk7 and Kin28 with Hint/PKCI-1 and Hnt1 histidine triad proteins," Journal of Biological Chemistry, vol. 275, no. 45, pp. 3483734840, 2000.

[19] E. Razin, Z. C. Zhang, H. Nechushtan et al., "Suppression of microphthalmia transcriptional activity by its association with protein kinase C-interacting protein 1 in mast cells," Journal of Biological Chemistry, vol. 274, no. 48, pp. 34272-34276, 1999.

[20] Y. N. Lee and E. Razin, "Nonconventional involvement of LysRS in the molecular mechanism of USF2 transcriptional activity in FceRI-activated mast cells," Molecular and Cellular Biology, vol. 25, no. 20, pp. 8904-8912, 2005.

[21] O. Huber and J. Weiske, “ $\beta$-catenin takes a HIT," Cell Cycle, vol. 7, no. 10, pp. 1326-1331, 2008.

[22] D. F. Calvisi, S. Ladu, F. Pinna et al., "SKP2 and CKS1 promote degradation of cell cycle regulators and are associated with hepatocellular carcinoma prognosis," Gastroenterology, vol. 137, no. 5, pp. 1816-1826.e10, 2009.

[23] H. Huang, X. Wei, X. Su et al., "Clinical significance of expression of Hint1 and potential epigenetic mechanism in gastric cancer," International Journal of Oncology, vol. 38, no. 6, pp. 1557-1564, 2011.

[24] Y. J. Zhang, H. Li, W. HC et al., "Silencing of Hint1, a novel tumor suppressor gene, by promoter hypermethylation in hepatocellular carcinoma," Cancer Letters, vol. 275, no. 2, pp. 277-284, 2009.

[25] Q. Liu, A. C. Puche, and J. B. Wang, "Distribution and expression of protein kinase $\mathrm{C}$ interactive protein (PKCI/HINT1) in mouse central nervous system (CNS)," Neurochemical Research, vol. 33, no. 7, pp. 1263-1276, 2008. 
[26] M. Baron, "Genetics of schizophrenia and the new millennium: progress and pitfalls," The American Journal of Human Genetics, vol. 68, no. 2, pp. 299-312, 2001.

[27] R. E. Straub, C. J. MacLean, F. A. O’Neill, D. Walsh, and K. S. Kendler, "Support for a possible schizophrenia vulnerability locus in region 5q22-31 in Irish families," Molecular Psychiatry, vol. 2, no. 2, pp. 148-155, 1997.

[28] S. M. Stahl, Stahl's Essential Psychopharmacology: Neuroscientific Basis and Practical Applications, Cambridge University Press, UK, 2013.

[29] J. van Os and S. Kapur, "Schizophrenia," The Lancet, vol. 374, no. 9690, pp. 635-645, 2009.

[30] M. P. Vawter, T. Barrett, C. Cheadle et al., "Application of cDNA microarrays to examine gene expression differences in schizophrenia," Brain Research Bulletin, vol. 55, no. 5, pp. 641-650, 2001.

[31] M. P. Vawter, J. M. Crook, T. M. Hyde et al., "Microarray analysis of gene expression in the prefrontal cortex in schizophrenia: a preliminary study," Schizophrenia Research, vol. 58, no. 1, pp. 11-20, 2002.

[32] M. P. Vawter, C. Shannon Weickert, E. Ferran et al., "Gene expression of metabolic enzymes and a protease inhibitor in the prefrontal cortex are decreased in schizophrenia," Neurochemical Research, vol. 29, no. 6, pp. 1245-1255, 2004.

[33] X. Chen, X. Wang, S. Hossain et al., "Haplotypes spanning SPEC2, PDZ-GEF2 and ACSL6 genes are associated with schizophrenia," Human Molecular Genetics, vol. 15, no. 22, pp. 3329-3342, 2006.

[34] J. Varadarajulu, A. Schmitt, P. Falkai, M. Alsaif, C. W. Turck, and D. Martins-de-Souza, "Differential expression of HINT1 in schizophrenia brain tissue," European Archives of Psychiatry and Clinical Neuroscience, vol. 262, no. 2, pp. 167-172, 2012.

[35] N. Kurotaki, S. Tasaki, H. Mishima et al., "Identification of novel schizophrenia loci by homozygosity mapping using DNA microarray analysis," PLoS One, vol. 6, no. 5, article e20589, 2011.

[36] E. Barbier, A. Zapata, E. Oh et al., "Supersensitivity to amphetamine in protein kinase-C interacting protein/HINT1 knockout mice," Neuropsychopharmacology, vol. 32, no. 8, pp. 1774-1782, 2007.

[37] C. A. Tamminga, "The neurobiology of cognition in schizophrenia," The Journal of Clinical Psychiatry, vol. 67, no. 9, article e11, 2006.

[38] M. van den Buuse, B. Garner, A. Gogos, and S. Kusljic, "Importance of animal models in schizophrenia research," Australian \& New Zealand Journal of Psychiatry, vol. 39, no. 7, pp. 550-557, 2005.

[39] Y. H. Dang, P. Liu, R. Ma et al., "HINT1 is involved in the behavioral abnormalities induced by social isolation rearing," Neuroscience Letters, vol. 607, pp. 40-45, 2015.

[40] B. J. Li, P. Liu, Z. Chu et al., "Social isolation induces schizophrenia-like behavior potentially associated with HINT1, NMDA receptor 1, and dopamine receptor 2," NeuroReport, vol. 28, no. 8, pp. 462-469, 2017.

[41] H. Skre, "Genetic and clinical aspects of Charcot-Marie-Tooth's disease," Clinical Genetics, vol. 6, no. 2, pp. 98-118, 1974.

[42] H. Zhao, V. Race, G. Matthijs et al., "Exome sequencing reveals HINT1 mutations as a cause of distal hereditary motor neuropathy," European Journal of Human Genetics, vol. 22, 2013.
[43] M. Zimoń, J. Baets, L. Almeida-Souza et al., "Loss-of-function mutations in HINT1 cause axonal neuropathy with neuromyotonia," Nature Genetics, vol. 44, no. 10, pp. 1080-1083, 2012.

[44] P. Maddison, "Neuromyotonia," Clinical Neurophysiology, vol. 117, no. 10, pp. 2118-2127, 2006.

[45] A. Horga, E. Cottenie, P. J. Tomaselli et al., "Absence of HINT1 mutations in a UK and Spanish cohort of patients with inherited neuropathies," Journal of Neurology, vol. 262, no. 8, pp. 1984-1986, 2015.

[46] N. U. Jerath, M. E. Shy, T. Grider, and L. Gutmann, "A case of neuromyotonia and axonal motor neuropathy: a report of a HINT1 mutation in the United States," Muscle \& Nerve, vol. 52, no. 6, pp. 1110-1113, 2015.

[47] P. Laššuthová, D. Š. Brožková, M. Krůtová et al., "Mutations in HINT1 are one of the most frequent causes of hereditary neuropathy among Czech patients and neuromyotonia is rather an underdiagnosed symptom," Neurogenetics, vol. 16, no. 1, pp. 43-54, 2015.

[48] K. L. Seburn, K. H. Morelli, A. Jordanova, and R. W. Burgess, "Lack of neuropathy-related phenotypes in Hint1 knockout mice," Journal of Neuropathology \& Experimental Neurology, vol. 73, no. 7, pp. 693-701, 2014.

[49] B. J. Kaplan, V. A. Sadock, and P. Ruiz, "Kaplan and Sadock's synopsis of psychiatry: behavioral sciences/clinical psychiatry," JAMA, vol. 290, no. 17, p. 2333, 2016.

[50] M. Elashoff, B. W. Higgs, R. H. Yolken et al., "Meta-analysis of 12 genomic studies in bipolar disorder," Journal of Molecular Neuroscience, vol. 31, no. 3, pp. 221-243, 2007.

[51] E. Barbier and J. B. Wang, "Anti-depressant and anxiolytic like behaviors in PKCI/HINT1 knockout mice associated with elevated plasma corticosterone level," BMC Neuroscience, vol. 10, p. 132, 2009.

[52] J. Garzon-Nino, M. Rodriguez-Munoz, E. Cortes-Montero, and P. Sanchez-Blazquez, "Increased PKC activity and altered GSK3 $\beta$ /NMDAR function drive behavior cycling in HINT1deficient mice: bipolarity or opposing forces," Scientific Reports, vol. 7, article 43468, 2017.

[53] D. Martins-de-Souza, P. C. Guest, L. W. Harris et al., "Identification of proteomic signatures associated with depression and psychotic depression in post-mortem brains from major depression patients," Translational Psychiatry, vol. 2, article e87, 2012.

[54] L. Ge, M. M. Zhu, J. Y. Yang et al., "Differential proteomic analysis of the anti-depressive effects of oleamide in a rat chronic mild stress model of depression," Pharmacology Biochemistry and Behavior, vol. 131, pp. 77-86, 2015.

[55] J. Varadarajulu, M. Lebar, G. Krishnamoorthy et al., "Increased anxiety-related behaviour in Hint1 knockout mice," Behavioural Brain Research, vol. 220, no. 2, pp. 305-311, 2011.

[56] K. J. Jackson, J. B. Wang, E. Barbier, X. Chen, and M. I. Damaj, "Acute behavioral effects of nicotine in male and female HINT1 knockout mice," Genes, Brain and Behavior, vol. 11, 2012.

[57] W. Guang, H. Wang, T. Su, I. B. Weinstein, and J. B. Wang, "Role of mPKCI, a novel $\mu$-opioid receptor interactive protein, in receptor desensitization, phosphorylation, and morphineinduced analgesia," Molecular Pharmacology, vol. 66, no. 5, pp. 1285-1292, 2004.

[58] F. Liu, J. Ma, P. Liu et al., "Hint1 gene deficiency enhances the supraspinal nociceptive sensitivity in mice," Brain and Behavior, vol. 6, no. 8, article e00496, 2016. 
[59] P. Sanchez-Blazquez, M. Rodriguez-Munoz, E. Berrocoso, and J. Garzon, "The plasticity of the association between muopioid receptor and glutamate ionotropic receptor $\mathrm{N}$ in opioid analgesic tolerance and neuropathic pain," European Journal of Pharmacology, vol. 716, no. 1-3, pp. 94-105, 2013.

[60] A. Vicente-Sanchez, P. Sanchez-Blazquez, M. RodriguezMunoz, and J. Garzon, "HINT1 protein cooperates with cannabinoid 1 receptor to negatively regulate glutamate NMDA receptor activity," Molecular Brain, vol. 6, p. 42, 2013.

[61] J. Garzon, R. Herrero-Labrador, M. Rodriguez-Munoz et al., "HINT1 protein: a new therapeutic target to enhance opioid antinociception and block mechanical allodynia," Neuropharmacology, vol. 89, pp. 412-423, 2015.

[62] P. Klepstad, T. Fladvad, F. Skorpen et al., "Influence from genetic variability on opioid use for cancer pain: a European genetic association study of 2294 cancer pain patients," Pain, vol. 152, no. 5, pp. 1139-1145, 2011.

[63] K. J. Jackson, Q. Chen, J. Chen, S. H. Aggen, K. S. Kendler, and $\mathrm{X}$. Chen, "Association of the histidine-triad nucleotidebinding protein-1 (HINT1) gene variants with nicotine dependence," The Pharmacogenomics Journal, vol. 11, no. 4, pp. 251-257, 2011.

[64] K. J. Jackson, J. B. Wang, E. Barbier, M. I. Damaj, and X. Chen, "The histidine triad nucleotide binding 1 protein is involved in nicotine reward and physical nicotine withdrawal in mice," Neuroscience Letters, vol. 550, pp. 129-133, 2013.

[65] R. Ray, C. Jepson, E. P. Wileyto et al., "Genetic variation in mu-opioid-receptor-interacting proteins and smoking cessation in a nicotine replacement therapy trial," Nicotine \& Tobacco Research, vol. 9, no. 11, pp. 1237-1241, 2007.

[66] E. V. Romanova, J. E. Lee, N. L. Kelleher, J. V. Sweedler, and J. M. Gulley, "Mass spectrometry screening reveals peptides modulated differentially in the medial prefrontal cortex of rats with disparate initial sensitivity to cocaine," The AAPS Journal, vol. 12, no. 3, pp. 443-454, 2010.

[67] D. A. Klein and J. M. Gulley, "Reduced sensitivity to the locomotor-stimulant effects of cocaine is associated with increased sensitivity to its discriminative stimulus properties," Behavioural Pharmacology, vol. 20, no. 1, pp. 67-77, 2009.

[68] J. Sabeti, G. A. Gerhardt, and N. R. Zahniser, "Individual differences in cocaine-induced locomotor sensitization in low and high cocaine locomotor-responding rats are associated with differential inhibition of dopamine clearance in nucleus accumbens," Journal of Pharmacology and Experimental Therapeutics, vol. 305, no. 1, pp. 180-190, 2003.

[69] R. M. Allen, C. V. Everett, A. M. Nelson, J. M. Gulley, and N. R. Zahniser, "Low and high locomotor responsiveness to cocaine predicts intravenous cocaine conditioned place preference in male Sprague-Dawley rats," Pharmacology Biochemistry and Behavior, vol. 86, no. 1, pp. 37-44, 2007.

[70] B. H. Mandt, S. Schenk, N. R. Zahniser, and R. M. Allen, "Individual differences in cocaine-induced locomotor activity in male Sprague-Dawley rats and their acquisition of and motivation to self-administer cocaine," Psychopharmacology, vol. 201, no. 2, pp. 195-202, 2008.

[71] J. P. Li, P. Liu, G. Lei et al., "The role of HINT1 in methamphetamine-induced conditioned place preference," Neurotoxicity Research, 2017.

[72] R. Weitzdoerfer, D. Stolzlechner, M. Dierssen, J. Ferreres, M. Fountoulakis, and G. Lubec, "Reduction of nucleoside diphosphate kinase B, Rab GDP- dissociation inhibitor beta and histidine triad nucleotide-binding protein in fetal Down syndrome brain," Protein Expression in Down Syndrome Brain, vol. 61, pp. 347-359, 2001.

[73] R. Abdel Rassoul, S. Alves, V. Pantesco et al., "Distinct transcriptome expression of the temporal cortex of the primate Microcebus murinus during brain aging versus Alzheimer's disease-like pathology," PLoS One, vol. 5, no. 9, 2010.

[74] K. M. Tye, "Neural circuit reprogramming: a new paradigm for treating neuropsychiatric disease?," Neuron, vol. 83, no. 6, pp. 1259-1261, 2014.

[75] S. K. Ajit, S. Ramineni, W. Edris et al., "RGSZ1 interacts with protein kinase $\mathrm{C}$ interacting protein PKCI-1 and modulates mu opioid receptor signaling," Cellular Signalling, vol. 19, no. 4, pp. 723-730, 2007.

[76] M. Rodriguez-Munoz, E. Cortes-Montero, A. PozoRodrigalvarez, P. Sanchez-Blazquez, and J. Garzon-Nino, "The ON:OFF switch, $\sigma 1 \mathrm{R}-\mathrm{HINT} 1$ protein, controls GPCRNMDA receptor cross-regulation: implications in neurological disorders," Oncotarget, vol. 6, no. 34, pp. 35458-35477, 2015.

[77] M. Rodriguez-Munoz, E. de la Torre-Madrid, P. SanchezBlazquez, and J. Garzon, "NO-released zinc supports the simultaneous binding of Raf- 1 and $\mathrm{PKC} \gamma$ cysteine-rich domains to HINT1 protein at the mu-opioid receptor," Antioxidants \& Redox Signaling, vol. 14, no. 12, pp. 2413-2425, 2011.

[78] M. Rodriguez-Munoz, E. de la Torre-Madrid, P. SanchezBlazquez, J. B. Wang, and J. Garzon, "NMDAR-nNOS generated zinc recruits PKC $\gamma$ to the HINT1-RGS17 complex bound to the $\mathrm{C}$ terminus of $\mathrm{Mu}$-opioid receptors," Cellular Signalling, vol. 20, no. 10, pp. 1855-1864, 2008.

[79] M. Rodriguez-Munoz, P. Sanchez-Blazquez, R. HerreroLabrador et al., "The $\sigma 1$ receptor engages the redoxregulated HINT1 protein to bring opioid analgesia under NMDA receptor negative control," Antioxidants \& Redox Signaling, vol. 22, no. 10, pp. 799-818, 2015.

[80] M. Rodriguez-Munoz, P. Sanchez-Blazquez, M. Merlos, and J. Garzon-Nino, "Endocannabinoid control of glutamate NMDA receptors: the therapeutic potential and consequences of dysfunction," Oncotarget, vol. 7, 2016.

[81] M. Rodriguez-Munoz, P. Sanchez-Blazquez, A. VicenteSanchez, C. Bailon, B. Martin-Aznar, and J. Garzon, "The histidine triad nucleotide-binding protein 1 supports mu-opioid receptor-glutamate NMDA receptor cross-regulation," Cellular and Molecular Life Sciences, vol. 68, no. 17, pp. 29332949, 2011.

[82] P. Sanchez-Blazquez, M. Rodriguez-Munoz, C. Bailon, and J. Garzon, "GPCRs promote the release of zinc ions mediated by nNOS/NO and the redox transducer RGSZ2 protein," Antioxidants \& Redox Signaling, vol. 17, no. 9, pp. 1163-1177, 2012.

[83] P. Sanchez-Blazquez, M. Rodriguez-Munoz, A. Vicente-Sanchez, and J. Garzon, "Cannabinoid receptors couple to NMDA receptors to reduce the production of NO and the mobilization of zinc induced by glutamate," Antioxidants \& Redox Signaling, vol. 19, no. 15, pp. 1766-1782, 2013.

[84] K. Yashiro and B. D. Philpot, "Regulation of NMDA receptor subunit expression and its implications for LTD, LTP, and metaplasticity," Neuropharmacology, vol. 55, no. 7, pp. 10811094, 2008.

[85] L. Sun, P. Liu, F. Liu et al., "Effects of Hint1 deficiency on emotional-like behaviors in mice under chronic immobilization stress," Brain and Behavior, vol. 7, 2017. 
[86] M. E. Greenberg, B. Xu, B. Lu, and B. L. Hempstead, "New insights in the biology of BDNF synthesis and release: implications in CNS function," Journal of Neuroscience, vol. 29, no. 41, pp. 12764-12767, 2009.

[87] D. Suri and V. A. Vaidya, "Glucocorticoid regulation of brain-derived neurotrophic factor: relevance to hippocampal structural and functional plasticity," Neuroscience, vol. 239, pp. 196-213, 2013. 

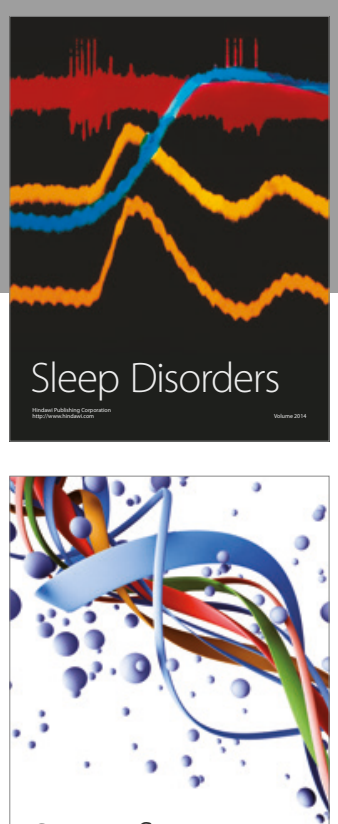

Scientifica
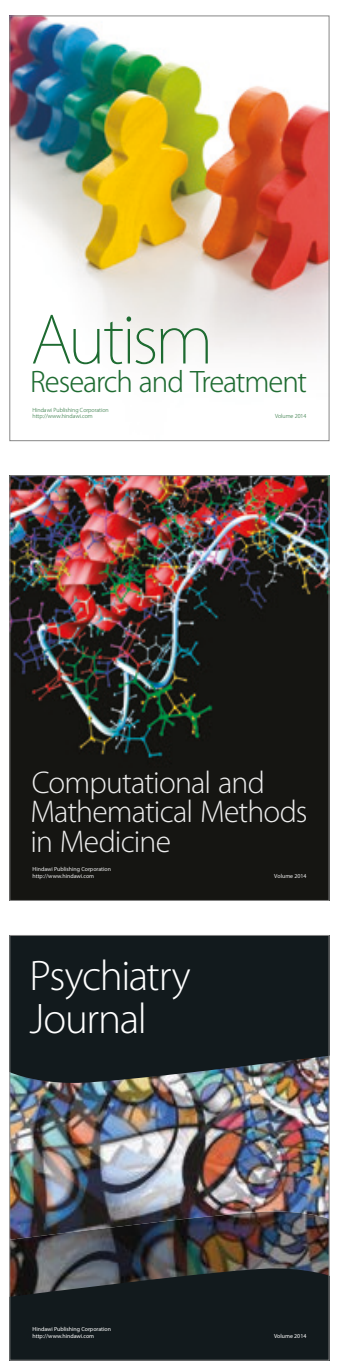
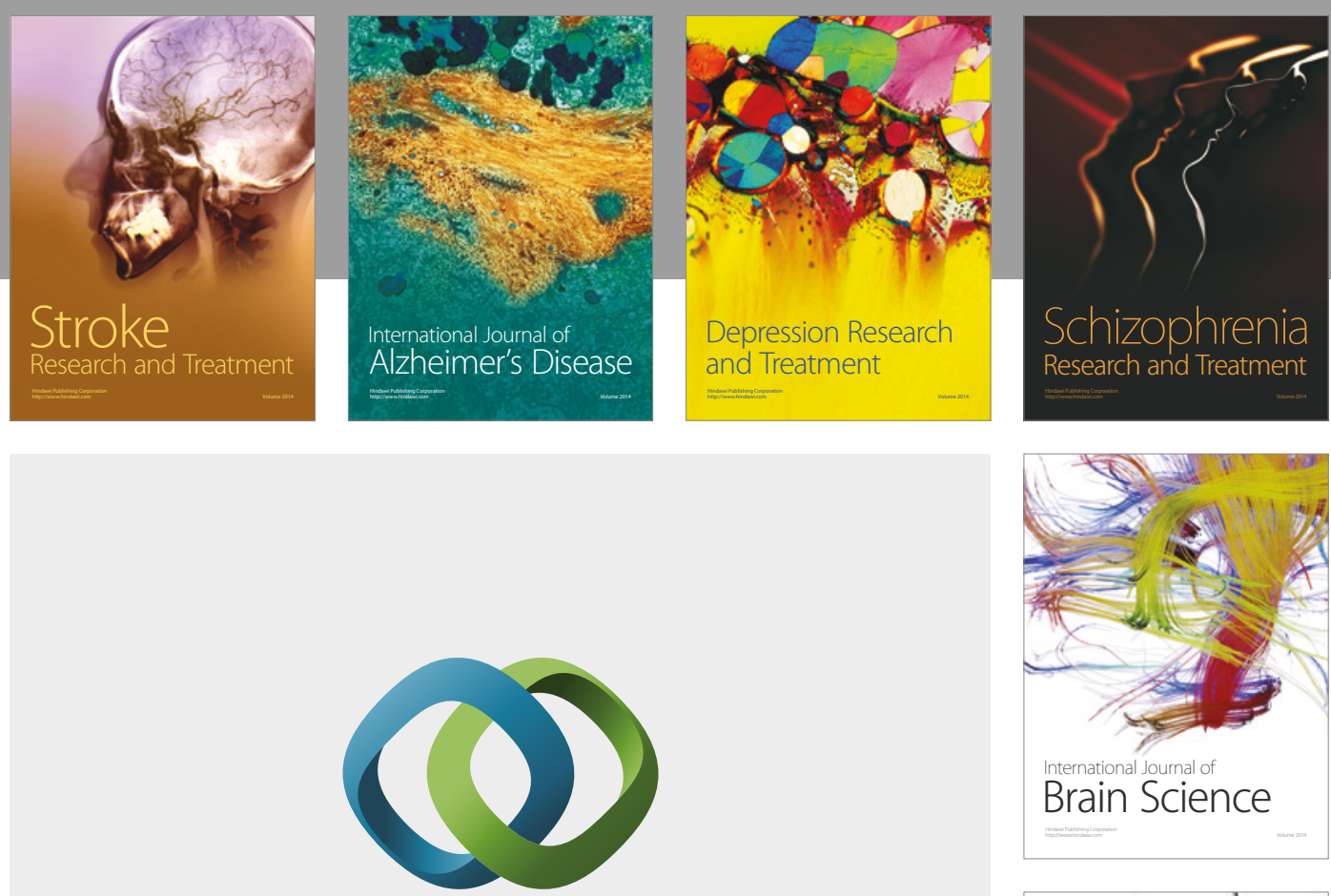

\section{Hindawi}

Submit your manuscripts at

https://www.hindawi.com
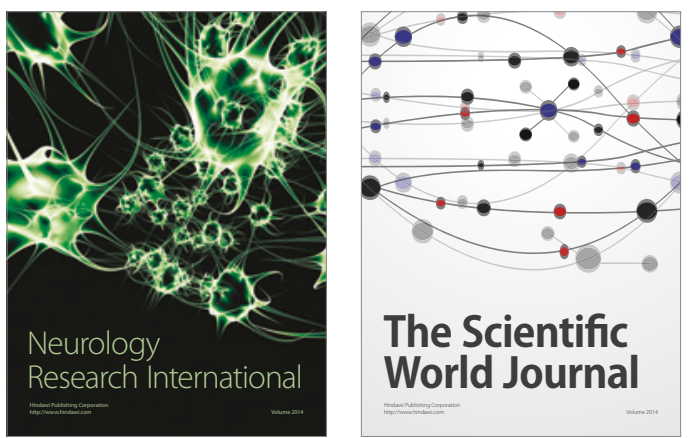

The Scientific World Journal

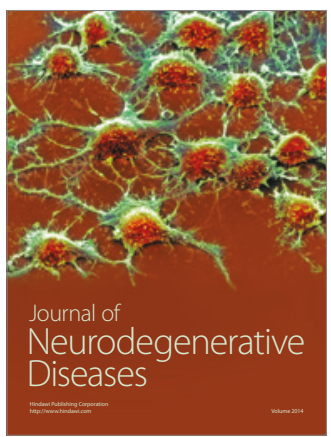

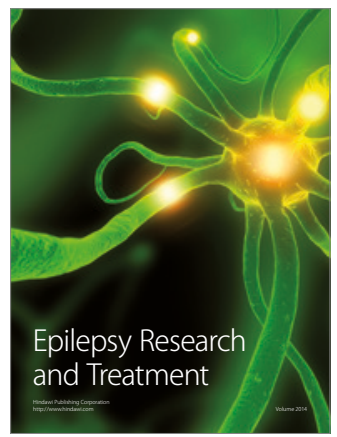

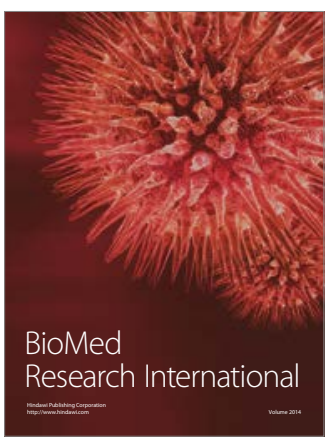

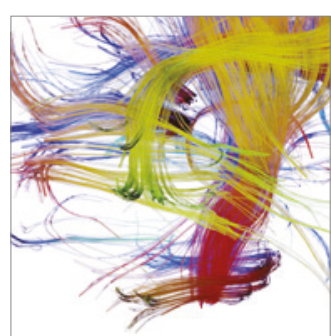

Brain Science

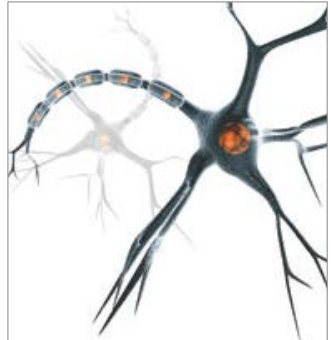

Neural Plasticity
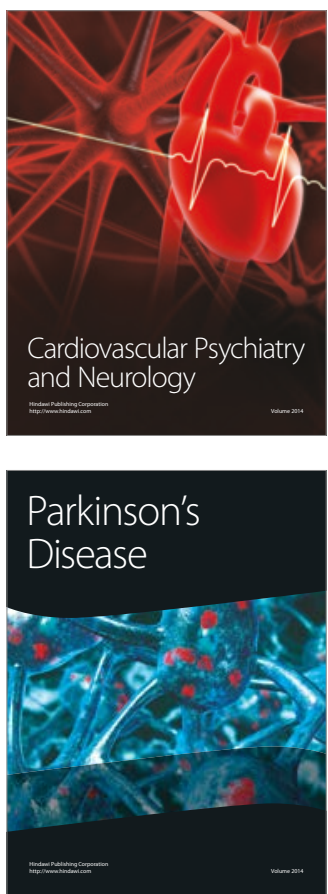